\title{
On decay and blow-up of solutions for a nonlinear beam equation with double damping terms
}

Jiali Yu', Yadong Shang ${ }^{1 *}$ and Huafei $\mathrm{Di}^{1}$

"Correspondence:

gzydshang@126.com

${ }^{1}$ School of Mathematics and

Information Science, Guangzhou

University, Guangzhou, P.R. China

\section{Springer}

\begin{abstract}
This paper deals with the initial boundary value problem for the nonlinear beam equation with double damping terms

$$
u_{t t}-u_{x x t}+u_{x x x x}+u_{x x x x t}=g\left(u_{x x}\right)_{x x,} \quad x \in \Omega, t>0
$$

where $\Omega=(0,1)$, and $g(s)$ is a given nonlinear function. We derive sufficient conditions for the blow-up of the solution to the problem by virtue of an adapted concavity method. In addition, global existence of weak solutions as well as exponential and uniform decay rates of the solution energy are established by the use of an integral inequality.
\end{abstract}

MSC: 35B44; 35K30; 35K59

Keywords: Nonlinear beam equation; Initial boundary value problem; Existence of weak solution; Blow-up; Energy decay

\section{Introduction}

In this paper, we study the following initial boundary value problem:

$$
\begin{aligned}
& u_{t t}-u_{x x t}+u_{x x x x}+u_{x x x x t}=g\left(u_{x x}\right)_{x x}, \quad x \in \Omega, t>0, \\
& u(0, t)=u(1, t)=0, \quad u_{x}(0, t)=u_{x}(1, t)=0, \quad t \geq 0, \\
& u(x, 0)=u_{0}(x) \in H_{0}^{2}(\Omega), \quad u_{t}(x, 0)=u_{1}(x) \in L^{2}(\Omega),
\end{aligned}
$$

where $u(x, t)$ denotes the unknown function, $g(s)$ is a given nonlinear function, $\Omega=(0,1)$, $u_{0}(x)$ and $u_{1}(x)$ are given functions which satisfy the boundary conditions (1.2), $u_{x x x x t}$ denotes the strong material damping, and $u_{x x t}$ represents the internal dynamic damping.

The system (1.1) models the motion of a neo-Hookean elastomer rod with internal damping, and as a model of a class of abstract nonlinear damped hyperbolic equations appears in many applications to natural sciences, such as the unidirectional propagation of nonlinear longitudinal displacement [1], dynamical longitudinal vibrations [2] and vibrations of a nonlinear damped beam [3].

(c) The Author(s) 2018. This article is distributed under the terms of the Creative Commons Attribution 4.0 International License (http://creativecommons.org/licenses/by/4.0/), which permits unrestricted use, distribution, and reproduction in any medium, provided you give appropriate credit to the original author(s) and the source, provide a link to the Creative Commons license, and indicate if changes were made. 
A general class of abstract evolution equations was established by Banks et al. [1]:

$$
\begin{aligned}
& w_{t t}+A_{1} w+A_{2} w_{t}+N^{*} g(N w)=f(t), \\
& w(0)=\varphi_{0}, \\
& w_{t}(0)=\varphi_{1},
\end{aligned}
$$

where $A_{2} w_{t}$ is the exact form of the internal dynamic damping mechanisms in elastomers, and $A_{1}, A_{2}, N$ and $f$ satisfy certain assumptions (see [1]). The global existence, uniqueness, regularity and continuous dependence on the initial data of a generalized solution to the problem (1.4)-(1.6) are proved under general conditions on the nonlinear term. This class contains problem (1.1)-(1.3) as a particular example. This example was discussed in a separate section in [1]. To obtain global existence, the authors of [1] used the Galerkin approximation method and the monotonicity method (see [4]). And in [3], the existence and uniqueness of weak solutions to a class of nonlinear beam equation are established under certain assumptions (locally Lipschitz plus affine domination) on the nonlinearity. Their results weaken the stringent monotonicity assumptions in the previous theories. In [5], the existence and uniqueness of the generalized global solutions and the classical global solutions of the three different initial boundary value problems for a damped nonlinear hyperbolic equation are proved by Chen. Moreover, sufficient conditions for a blow-up of solutions are given in [5].

In 2010, Song et al. [6] proved the existence and nonexistence of global solutions to the beam equation

$$
u_{t t}+k_{1} u_{x x x x}+k_{2} u_{x x x x t}=g\left(u_{x x}\right)_{x x}
$$

with the initial value conditions

$$
u(x, 0)=u_{0}(x), \quad u_{t}(x, 0)=u_{1}(x), \quad x \in \mathbb{R} .
$$

Applying the Fourier transform method, the authors proved that for any $T>0$, the Cauchy problem (1.7)-(1.8) admits a unique global smooth solution $u \in C^{\infty}\left((0, T] ; H^{\infty}(\mathbb{R})\right) \cap$ $C\left((0, T] ; H^{3}(\mathbb{R})\right) \cap C^{1}\left((0, T] ; H^{-1}(\mathbb{R})\right)$ in the case of $g(s)=s^{n}$. Recently, Yu et al. [7] studied the following wave equation:

$$
u_{t t}-k_{1} u_{x x t}+u_{x x x x}+u_{x x x x t}=g\left(u_{x}\right)_{x}, \quad x \in \mathbb{R}, t>0,
$$

with the initial value conditions (1.8). They gave sufficient conditions on the blow-up of the solution in finite time and an example.

In [8], Chen et al. gave sufficient conditions of a blow-up of the solutions for the problem:

$$
\begin{aligned}
& u_{t t}+k_{1} \Delta^{2} u+k_{2} \Delta^{2} u_{t}+\Delta g(\Delta u)=0, \quad x \in \Omega, t>0, \\
& u=0, \quad \frac{\partial u}{\partial v}=0, \quad x \in \partial \Omega, t \geq 0, \\
& u(x, 0)=u_{0}(x), \quad u_{t}(x, 0)=u_{1}(x), \quad x \in \bar{\Omega},
\end{aligned}
$$


and proved the existence and uniqueness of the local generalized solution for this problem. Furthermore, Aassila and Guesmia in [9] solved the energy decay problem for $\Omega \subset \mathbb{R}^{m}$. Later on, their results were extended to various problems, for more investigations and different points of view we refer to [10-21] and the references therein.

There have been many impressive works on the well-posedness and energy decay of solutions to the nonlinear beam equations of the type (1.1). However, to the best of our knowledge, various authors considered the problem driven only by either material damping or nonlinear evolution equation with dynamic damping term, see [1-6, 8-12]. There is little research on the well-posedness and energy decay of Eq. (1.1) with material damping and internal dynamic damping at the same time. For this kind of equation, the questions of the existence and nonexistence of global generalized solutions (or the global weak solutions) to the Cauchy problem and the initial boundary value problem remain open.

In the present paper, we study the existence and uniqueness of the global weak solution for the initial boundary value problem (1.1)-(1.3). And then we focus on finding sufficient conditions yielding nonexistence of a global solution to the above problem. We construct a differential inequality under certain conditions. The method applied is the so-called "concavity method" [21]. As an application of the above results, one example of nonexistence of a global solution is given. Our methods for studying the blow-up of solution are applicable to other nonlinear evolution equations. Finally, we study the asymptotic behavior of the solution to the problem (1.1)-(1.3) by the use of an integral inequality.

This paper is organized as follows. In Sect. 2, some notations and the main results are stated. In Sect. 3, the existence of global weak solutions to problem (1.1)-(1.3) is studied. Section 4 contains the statement and the proofs of the blow-up of solutions. Section 5 gives the statement and the proofs of the decay estimate of the energy functional. In Sect. 6, a comparison of various results is carried out. And we also state some open and significant questions.

\section{Preliminaries and main results}

In this section, some notations and the main results for the initial boundary value problem (1.1)-(1.3) are stated. Letting $L^{2}=L^{2}(\Omega)$ and $H=H_{0}^{2}(\Omega)$, we have the Gelfand triple $H \hookrightarrow$ $L^{2} \hookrightarrow H^{*}$ with $H^{*}=H^{-2}(\Omega)$.

For brevity, we use the following abbreviations:

$$
\|\cdot\|=\|\cdot\|_{L^{2}(\Omega)}, \quad\|\cdot\|_{H}=\|\cdot\|_{H_{0}^{2}(\Omega)}, \quad\|\cdot\|_{H^{*}}=\|\cdot\|_{H^{-2}(\Omega)} \cdot
$$

Also $(\cdot, \cdot)$ denotes the $L^{2}$-inner product, while $(\cdot, \cdot)_{H^{*}, H}$ stands for the usual duality product. Assume that the parameters in (1.1)-(1.3) satisfy the following assumptions:

Assumption 2.1 We assume that

$$
G(\xi)=\int_{0}^{\xi} g(\tau) d \tau, \quad g(\xi)=G^{\prime}(\xi)
$$

satisfy the following conditions:

(a) There exist positive constants $C_{i}$ for $i=1,2,3$ such that

$$
-C_{2}|\xi|^{2}-C_{3} \leq G(\xi) \leq \frac{1}{2}(1-\varepsilon)|\xi|^{2}+C_{1}, \quad \forall \varepsilon>0 .
$$


(b) There are positive constants $\widetilde{C}_{j}$ for $j=1,2$ such that

$$
|g(\xi)| \leq \widetilde{C}_{1}|\xi|+\widetilde{C}_{2}
$$

(c) $g$ is a nondecreasing function, i.e.,

$$
g^{\prime}(\xi) \geq 0, \quad \forall \xi \in \mathbb{R}
$$

Definition 2.1 We denote the function space

$$
X(T)=\left\{w \mid w \in L^{2}(0, T ; H), w_{t} \in L^{2}(0, T ; H), w_{t t} \in L^{2}\left(0, T ; H^{*}\right)\right\}
$$

equipped with the norm

$$
\|w\|_{X(T)}=\left(\|w\|_{L^{2}(0, T ; H)}^{2}+\left\|w_{t}\right\|_{L^{2}(0, T ; H)}^{2}+\left\|w_{t t}\right\|_{L^{2}\left(0, T ; H^{*}\right)}^{2}\right)^{\frac{1}{2}} .
$$

We now define the concept of a weak solution to the problem (1.1)-(1.3).

Definition 2.2 A function $u \in X(T)$ is a global weak solution of (1.1)-(1.3) if it satisfies the following identity for every $t \in[0, T]$

$$
\begin{aligned}
& \left(u_{t t}(t), v\right)_{H^{*}, H}-\left(u_{t}(t), v_{x x}\right)+\left(u_{x x}(t), v_{x x}\right)+\left(u_{x x t}(t), v_{x x}\right) \\
& \quad=\left(g\left(u_{x x}(t)\right), v_{x x}\right)
\end{aligned}
$$

for all $v \in H$. In addition, $u$ must also satisfy

$$
u(x, 0)=u_{0}(x) \in H, \quad u_{t}(x, 0)=u_{1}(x) \in L^{2} .
$$

For the problem (1.1)-(1.3), we have the following theorem.

Theorem 2.1 Suppose that $u_{0}(x) \in H, u_{1}(x) \in L^{2}$ and that Assumption 2.1 holds. Then the problem (1.1)-(1.3) has a global weak solution $u \in X(T)$.

Theorem 2.2 Suppose that $g \in C^{2}(\mathbb{R}), u_{0} \in H^{2}(\Omega), u_{1} \in L^{2}(\Omega), G\left(u_{0 x x}\right) \in L^{1}(\Omega)$ and there exists a constant $\gamma>0$ such that

$$
s g(s) \geq 2(1+2 \gamma) G(s), \quad s \in \mathbb{R},
$$

where $G(s)=\int_{0}^{s} g(\tau) d \tau$.

Then the solution $u(x, t)$ of the problem (1.1)-(1.3) blows up in finite time if one of the following conditions holds true:

(1) $E(0)<0$;

(2) $E(0)=0,2\left(u_{0}, u_{1}\right)>\frac{1}{\gamma}\left(\left\|u_{0 x x}\right\|^{2}+\left\|u_{0 x}\right\|^{2}\right)$;

(3) $E(0)>0,\left(u_{0}, u_{1}\right)>\left[E(0)\left(\left\|u_{0}\right\|^{2}+T_{0}\left\|u_{0 x}\right\|^{2}+T_{0}\left\|u_{0 x x}\right\|^{2}\right)\right]^{\frac{1}{2}}$, where $E(0)=\left\|u_{1}\right\|^{2}+\left\|u_{0 x x}\right\|^{2}-2 \int_{\Omega} G\left(u_{0 x x}\right) d x$. 
Theorem 2.3 Suppose that the conditions of Theorem 2.1 hold. Let $u(x, t) \in X(T)$ be a global weak solution of the problem (1.1)-(1.3). We also assume that

(1) $G(s) \leq 0$, for all $s \in \mathbb{R}$,

(2) $\operatorname{sg}(s) \leq 2 G(s)$, for all $s \in \mathbb{R}$.

Then

$$
E_{1}(t) \leq E_{1}(0) e^{1-\frac{t}{3}}
$$

where $E_{1}(t)=\left\|u_{t}(t)\right\|^{2}+\left\|u_{x x}(t)\right\|^{2}-2 \int_{0}^{1} G\left(u_{x x}\right) d x$.

\section{Existence of solution}

In this section, we establish the existence results for the problem (1.1)-(1.3) under suitable conditions. The method which we use is a Galerkin approximation similar to those used in [3] and [4].

Proof of Theorem 2.1 Let $\left\{\omega_{j}(x)\right\}$ be a system of basis functions in $H$. Now we construct the following approximate solution $u_{m}(x, t)$ of problem (1.1)-(1.3):

$$
u_{m}(x, t)=\sum_{j=1}^{m} \alpha_{j}^{m}(t) \omega_{j}(x), \quad m=1,2, \ldots
$$

which satisfies

$$
\begin{gathered}
\left(u_{m t t}(t), \omega_{i}\right)-\left(u_{m t}(t), \omega_{i x x}\right)+\left(u_{m x x}(t), \omega_{i x x}\right)+\left(u_{m x x t}(t), \omega_{i x x}\right) \\
=\left(g\left(u_{m x x}(t)\right), \omega_{i x x}\right), \quad i=1,2, \ldots, m,
\end{gathered}
$$

with initial conditions

$$
\begin{aligned}
& u_{m}(x, 0)=\sum_{j=1}^{m} a_{j}^{m} \omega_{j}(x) \rightarrow u_{0}(x) \quad \text { in } H \text { as } m \rightarrow+\infty, \\
& u_{m t}(x, 0)=\sum_{j=1}^{m} b_{j}^{m} \omega_{j}(x) \rightarrow u_{1}(x) \quad \text { in } L^{2} \text { as } m \rightarrow+\infty .
\end{aligned}
$$

Multiplying (3.1) by $\frac{d}{d t} \alpha_{i}^{m}(t)$ and summing over $i$, we obtain

$$
\begin{aligned}
& \left(u_{m t t}(t), u_{m t}(t)\right)-\left(u_{m t}(t), u_{m x x t}(t)\right)+\left(u_{m x x}(t), u_{m x x t}(t)\right)+\left(u_{m x x t}(t), u_{m x x t}(t)\right) \\
& \quad=\left(g\left(u_{m x x}(t)\right), u_{m x x t}(t)\right) .
\end{aligned}
$$

Hence,

$$
\begin{aligned}
& \frac{d}{d t}\left[\left\|u_{m t}(t)\right\|^{2}+\left\|u_{m x x}(t)\right\|^{2}\right]+2\left\|u_{m x t}(t)\right\|^{2}+2\left\|u_{m x x t}(\cdot, t)\right\|^{2} \\
& \quad=2\left(g\left(u_{m x x}(t)\right), u_{m x x}(t)\right) .
\end{aligned}
$$


From Assumption 2.1 and (3.5), we have

$$
\begin{aligned}
& \frac{d}{d t}\left[\left\|u_{m t}(t)\right\|^{2}+\left\|u_{m x x}(t)\right\|^{2}-2 \int_{0}^{1} G\left(u_{x x}\right) d x\right] \\
& \quad+2\left\|u_{m x t}(t)\right\|^{2}+2\left\|u_{m x x t}(t)\right\|^{2}=0 .
\end{aligned}
$$

Using this fact we conclude that there exists a positive constant $C$ independent on $m$ such that

$$
\begin{gathered}
\left\|u_{m t}(t)\right\|^{2}+\varepsilon\left\|u_{m x x}(t)\right\|^{2}+2 \int_{0}^{t}\left\|u_{m x \tau}(\tau)\right\|^{2} d \tau \\
+2 \int_{0}^{t}\left\|u_{m x x \tau}(\tau)\right\|^{2} d \tau \leq C, \quad t \in[0, T] .
\end{gathered}
$$

Therefore, there exist limit functions $u(x, t) \in W^{1,2}(0, T ; H)$ and a subsequence still denoted $\left\{u_{m}\right\}$, for which as $m \rightarrow+\infty$ we have

$$
u_{m} \rightarrow u \quad \text { weakly in } W^{1,2}(0, T ; H) .
$$

From Assumption 2.1(b), we have

$$
\begin{aligned}
& \left\|g\left(u_{m x x}\right)\right\|_{L^{2}\left(0, T ; L^{2}\right)}^{2} \\
& \quad=\int_{0}^{t} \int_{0}^{1}\left|g\left(u_{m x x}(x, \tau)\right)\right|^{2} d x d \tau \\
& \quad \leq 2 \int_{0}^{t} \int_{0}^{1}\left(\tilde{C}_{1}^{2}\left|u_{m x x}(x, \tau)\right|^{2}+\tilde{C}_{2}^{2}\right) d x d \tau \\
& =2 \tilde{C}_{1}^{2} \int_{0}^{t}\left\|u_{m x x}(\tau)\right\|^{2} d \tau+2 \tilde{C}_{2}^{2} t \\
& \quad \leq 2 \tilde{C}_{1}^{2} C t+2 \tilde{C}_{2}^{2} t \leq 2\left(\tilde{C}_{1}^{2} C+\tilde{C}_{2}^{2}\right) T .
\end{aligned}
$$

Therefore, there exists a function $\tilde{g}(t) \in L^{2}\left(0, T ; L^{2}\right)$ for all $t \in[0, T]$ such that

$$
g\left(u_{m x x}(t)\right) \rightarrow \tilde{g}(t) \quad \text { weakly in } L^{2}\left(0, T ; L^{2}\right)
$$

In (3.1), we fix $i$ and let $m \rightarrow+\infty$. Then, we have

$$
\begin{aligned}
& \left(u_{t t}(t), \omega_{i}\right)-\left(u_{t}(t), \omega_{i x x}\right)+\left(u_{x x}(t), \omega_{i x x}\right)+\left(u_{x x t}(t), \omega_{i x x}\right) \\
& \quad=\left(\tilde{g}(t), \omega_{i x x}\right)
\end{aligned}
$$

and

$$
\begin{aligned}
& \left(u_{t t}(t), v\right)-\left(u_{t}(t), v_{x x}\right)+\left(u_{x x}(t), v_{x x}\right)+\left(u_{x x t}(t), v_{x x}\right) \\
& \quad=\left(\tilde{g}(t), v_{x x}\right), \quad \forall v \in H,
\end{aligned}
$$

with $u(x, 0)=u_{0}(x)$ and $u_{t}(x, 0)=u_{1}(x)$. 
From Assumption 2.1(c), following ideas in [4], we can prove that

$$
g\left(u_{x x}(t)\right)=\tilde{g}(t) \quad \text { for a.e. } t \in[0, T]
$$

By density we obtain that $u(x, t)$ is a weak solution to the problem (1.1)-(1.3).

We also note that

$$
\begin{aligned}
\nu \rightarrow\left(u_{t t}, v\right)_{H^{*}, H}= & -\int_{0}^{t}\left(u_{\tau}(\tau), v_{\tau}(\tau)\right) d \tau \\
= & \int_{0}^{t}\left(g\left(u_{x x}(\tau)\right), v_{x x}(\tau)\right) d \tau-\int_{0}^{t}\left(u_{x x}(\tau), v_{x x}(\tau)\right) d \tau \\
& -\int_{0}^{t}\left(u_{x x \tau}(\tau), v_{x x}(\tau)\right) d \tau+\int_{0}^{t}\left(u_{\tau}(\tau), v_{x x}(\tau)\right) d \tau
\end{aligned}
$$

is continuous in $\mathcal{D}(0, T ; H)$ equipped with the topology of $L^{2}(0, T ; H)$ and thus by density in $L^{2}(0, T ; H)$. So $u_{t t} \in L^{2}(0, T ; H)^{*}=L^{2}\left(0, T ; H^{*}\right)$. Since we have already established that $u(x, t) \in W^{1,2}(0, T ; H)$, we have that $u(x, t) \in X(T)$, and we obtain the additional regularity $u(x, t) \in C(0, T ; H)$ and $u_{t}(x, t) \in C\left(0, T ; L^{2}\right)$ by [22]. This completes the proof.

\section{Blow-up of solution}

In this section, we will prove a fact for the blow-up of solution to problem (1.1)-(1.3). The main method employed in this paper is based on the concavity argument developed by Levin $[23,24]$. In order to prove our main result, we will use the following lemma.

Lemma 4.1 ([21]) Suppose that a positive, twice-differentiable function $\theta(t)$ satisfies the inequality

$$
\theta^{\prime \prime}(t) \theta(t)-(1+\gamma) \theta^{\prime 2}(t) \geq-2 C_{1} \theta^{\prime}(t) \theta(t)-C_{2} \theta^{2}(t), \quad t>0
$$

where $\gamma>0$ and $C_{1}, C_{2} \geq 0$ are constants.

(1) If $C_{1}=C_{2}=0, \theta(0)>0$ and $\theta^{\prime}(0)>0$, then there exists a $t_{1} \in\left(0, \frac{\theta(0)}{\gamma \theta^{\prime}(0)}\right]$ such that $\theta(t)$ tends to infinity as $t \rightarrow t_{1}$.

(2) If $C_{1}+C_{2}>0, \theta(0)>0$ and $\theta^{\prime}(0)>-\gamma_{2} \gamma^{-1} \theta(0)$, then there exists a $t_{1}>0$ such that $\theta(t)$ tends to infinity as $t \rightarrow t_{1}$, where $t_{1}$ is bounded above by

$$
\begin{gathered}
\frac{1}{2 \sqrt{C_{1}^{2}+\gamma C_{2}}} \ln \frac{\gamma_{1} \theta(0)+\gamma \theta^{\prime}(0)}{\gamma_{2} \theta(0)+\gamma \theta^{\prime}(0)} \\
\text { with } \gamma_{1}=-C_{1}+\sqrt{C_{1}^{2}+\gamma C_{2}} \text { and } \gamma_{2}=-C_{1}-\sqrt{C_{1}^{2}+\gamma C_{2}} .
\end{gathered}
$$

Proof of Theorem 2.2 Suppose that the maximal time of existence of solution to the initial boundary value problem (1.1)-(1.3) is infinite. The energy functional can be defined as

$$
\begin{aligned}
E(t)= & \left\|u_{t}(t)\right\|^{2}+\left\|u_{x x}(t)\right\|^{2}+2 \int_{0}^{t}\left\|u_{x t}(\tau)\right\|^{2} d \tau \\
& +2 \int_{0}^{t}\left\|u_{x x t}(\tau)\right\|^{2} d \tau-2 \int_{\Omega} G\left(u_{x x}\right) d x .
\end{aligned}
$$


From (4.1) and (1.1), it follows that

$$
\begin{aligned}
\frac{d}{d t} E(t)= & \frac{d}{d t}\left[\left\|u_{t}(t)\right\|^{2}+\left\|u_{x x}(t)\right\|^{2}+2 \int_{0}^{t}\left\|u_{x t}(\tau)\right\|^{2} d \tau\right. \\
& \left.+2 \int_{0}^{t}\left\|u_{x x t}(\tau)\right\|^{2} d \tau-2 \int_{\Omega} G\left(u_{x x}\right) d x\right] \\
= & 2 \int_{\Omega}\left(u_{t t} u_{t}+u_{x x x x} u_{t}-u_{x x t} u_{t}+u_{x x x x t} u_{t}-g\left(u_{x x}\right)_{x x} u_{t}\right) d x \\
= & 0 .
\end{aligned}
$$

Integration of (4.2) from 0 to $t$ leads to

$$
E(t)=E(0)
$$

We now define

$$
\begin{aligned}
\phi(t)= & \|u(t)\|^{2}+\int_{0}^{t}\left\|u_{x}(\tau)\right\|^{2} d \tau+\int_{0}^{t}\left\|u_{x x}(\tau)\right\|^{2} d \tau \\
& +\left(T_{0}-t\right)\left\|u_{0 x}\right\|^{2}+\left(T_{0}-t\right)\left\|u_{0 x x}\right\|^{2}+\beta\left(t+t_{0}\right)^{2},
\end{aligned}
$$

where $0 \leq t \leq T_{0}, \beta$ and $t_{0}$ are nonnegative real numbers to be given later. Hence,

$$
\begin{aligned}
\phi^{\prime}(t)= & 2 \int_{\Omega} u(t) u_{t}(t) d x+\left\|u_{x}(t)\right\|^{2}+\left\|u_{x x}(t)\right\|^{2}-\left\|u_{0 x}\right\|^{2} \\
& -\left\|u_{0 x x}\right\|^{2}+2 \beta\left(t+t_{0}\right) \\
= & 2\left[\int_{\Omega} u(t) u_{t}(t) d x+\int_{0}^{t} \int_{\Omega} u_{x}(\tau) u_{x t}(\tau) d x d \tau\right. \\
& \left.+\int_{0}^{t} \int_{\Omega} u_{x x}(\tau) u_{x x t}(\tau) d x d \tau+\beta\left(t+t_{0}\right)\right]
\end{aligned}
$$

From (4.4) and (1.1), we have

$$
\begin{aligned}
\phi^{\prime \prime}(t) & =2\left[\left\|u_{t}(t)\right\|^{2}+\int_{\Omega} u(t) u_{t t}(t) d x+\int_{\Omega} u_{x}(t) u_{x t}(t) d x+\int_{\Omega} u_{x x}(t) u_{x x t}(t) d x+\beta\right] \\
& =2\left[\left\|u_{t}(t)\right\|^{2}+\int_{\Omega} u(t)\left[u_{t t}(t)-u_{x x t}(t)+u_{x x x x t}(t)\right] d x+\beta\right] \\
& =2\left[\left\|u_{t}(t)\right\|^{2}+\int_{\Omega} u(t)\left[-u_{x x x x}(t)+g\left(u_{x x}(t)\right)_{x x}\right] d x+\beta\right] \\
& =2\left[\left\|u_{t}(t)\right\|^{2}-\left\|u_{x x}(t)\right\|^{2}+\left(g\left(u_{x x}\right), u_{x x}\right)+\beta\right] .
\end{aligned}
$$

From (4.4), we can write

$$
\begin{aligned}
\phi^{\prime}(t)^{2}= & 4\left[\int_{\Omega} u(t) u_{t}(t) d x+\int_{0}^{t} \int_{\Omega} u_{x}(\tau) u_{x t}(\tau) d x d \tau+\int_{0}^{t} \int_{\Omega} u_{x x}(\tau) u_{x x t}(\tau) d x d \tau\right. \\
& \left.+\beta\left(t+t_{0}\right)\right]^{2}
\end{aligned}
$$




$$
\begin{aligned}
\leq & 4\left[\|u(t)\|\left\|u_{t}(t)\right\|+\left(\int_{0}^{t}\left\|u_{x}(\tau)\right\|^{2} d \tau\right)^{\frac{1}{2}}\left(\int_{0}^{t}\left\|u_{x t}(\tau)\right\|^{2} d \tau\right)^{\frac{1}{2}}\right. \\
& \left.+\left(\int_{0}^{t}\left\|u_{x x}(\tau)\right\|^{2} d \tau\right)^{\frac{1}{2}}\left(\int_{0}^{t}\left\|u_{x x t}(\tau)\right\|^{2} d \tau\right)^{\frac{1}{2}}+\sqrt{\beta} \sqrt{\beta}\left(t+t_{0}\right)\right]^{2} \\
\leq & 4 \phi(t)\left[\left\|u_{t}(t)\right\|^{2}+\int_{0}^{t}\left\|u_{x t}(\tau)\right\|^{2} d \tau+\int_{0}^{t}\left\|u_{x x t}(\tau)\right\|^{2} d \tau+\beta\right] .
\end{aligned}
$$

Now, from (4.4)-(4.6), we obtain

$$
\begin{aligned}
\phi^{\prime \prime}(t) \phi(t)-(1+\gamma) \phi^{\prime}(t)^{2} \\
=2 \phi(t)\left[\left\|u_{t}(t)\right\|^{2}-\left\|u_{x x}(t)\right\|^{2}+\int_{\Omega} g\left(u_{x x}(t)\right) u_{x x}(t) d x+\beta\right]-(1+\gamma) \phi^{\prime}(t)^{2} \\
\geq 2 \phi(t)\left[\left\|u_{t}(t)\right\|^{2}-\left\|u_{x x}(t)\right\|^{2}+\int_{\Omega} g\left(u_{x x}(t)\right) u_{x x}(t) d x+\beta\right. \\
\left.\quad-2(1+\gamma)\left(\left\|u_{t}(t)\right\|^{2}+\int_{0}^{t}\left\|u_{x t}(\tau)\right\|^{2} d \tau+\int_{0}^{t}\left\|u_{x x t}(\tau)\right\|^{2} d \tau+\beta\right)\right] \\
\geq 2 \phi(t) \psi(t),
\end{aligned}
$$

where

$$
\begin{aligned}
\psi(t)= & -\left\|u_{x x}(t)\right\|^{2}+\int_{\Omega} g\left(u_{x x}(t)\right) u_{x x}(t) d x-(2 \gamma+1)\left(\left\|u_{t}(t)\right\|^{2}+\beta\right) \\
& -2(1+\gamma) \int_{0}^{t}\left(\left\|u_{x t}(\tau)\right\|^{2}+\left\|u_{x x t}(\tau)\right\|^{2}\right) d \tau .
\end{aligned}
$$

From (4.8) and (1.1), we deduce

$$
\begin{aligned}
\psi^{\prime}(t)= & -2 \int_{\Omega} u_{x x}(t) u_{x x t}(t) d x+\frac{d}{d t} \int_{\Omega} g\left(u_{x x}(t)\right) u_{x x}(t) d x \\
& -2(2 \gamma+1) \int_{\Omega} u_{t}(t) u_{t t}(t) d x-2(1+\gamma)\left(\left\|u_{x t}(t)\right\|^{2}+\left\|u_{x x t}(t)\right\|^{2}\right) \\
= & -2 \int_{\Omega} u_{x x}(t) u_{x x t}(t) d x+\frac{d}{d t} \int_{\Omega} g\left(u_{x x}(t)\right) u_{x x}(t) d x \\
& -2(2 \gamma+1) \int_{\Omega} u_{t}(t)\left[u_{x x t}(t)-u_{x x x x}(t)-u_{x x x x t}(t)+g\left(u_{x x}(t)\right)_{x x}\right] d x \\
& -2(1+\gamma)\left(\left\|u_{x t}(t)\right\|^{2}+\left\|u_{x x t}(t)\right\|^{2}\right) \\
= & -2 \int_{\Omega} u_{x x}(t) u_{x x t}(t) d x+\frac{d}{d t} \int_{\Omega} g\left(u_{x x}(t)\right) u_{x x}(t) d x \\
& +2(2 \gamma+1) \int_{\Omega} u_{x x}(t) u_{x x t}(t) d x+2 \gamma\left(\left\|u_{x t}(t)\right\|^{2}+\left\|u_{x x t}(t)\right\|^{2}\right) \\
& -2(2 \gamma+1) \int_{\Omega} g\left(u_{x x}(t)\right) u_{x x t}(t) d x \\
& \frac{d}{d t} \int_{\Omega} g\left(u_{x x}(t)\right) u_{x x}(t) d x-2(2 \gamma+1) \int_{\Omega} g\left(u_{x x}(t)\right) u_{x x t}(t) d x \\
& +4 \gamma \int_{\Omega} u_{x x t}(t) u_{x x}(t) d x+2 \gamma\left\|u_{x x t}(t)\right\|^{2}+2 \gamma\left\|u_{x t}(t)\right\|^{2} . \\
& \\
&
\end{aligned}
$$


Integrating (4.9) from 0 to $t$ and making use of the assumption (2.1),

$$
\begin{aligned}
\psi(t) \geq & \psi(0)-\int_{\Omega} g\left(u_{0 x x}\right) u_{0 x x} d x+\int_{\Omega} g\left(u_{x x}(t)\right) u_{x x}(t) d x \\
& -2(1+2 \gamma) \int_{\Omega} G\left(u_{x x}(t)\right) d x+2(1+2 \gamma) \int_{\Omega} G\left(u_{0 x x}\right) d x \\
& +2 \gamma\left\|u_{x x}(t)\right\|^{2}-2 \gamma\left\|u_{0 x x}\right\|^{2} \\
\geq & \psi(0)-\int_{\Omega} g\left(u_{0 x x}\right) u_{0 x x} d x+2(1+2 \gamma) \int_{\Omega} G\left(u_{0 x x}\right) d x-2 \gamma\left\|u_{0 x x}\right\|^{2} \\
\geq & -(1+2 \gamma)\left(\left\|u_{0 x x}\right\|^{2}+\left\|u_{1}\right\|^{2}-2 \int_{\Omega} G\left(u_{0 x x}\right) d x+\beta\right) \\
\geq & -(1+2 \gamma)(E(0)+\beta) .
\end{aligned}
$$

Combining this inequality with (4.7), we obtain

$$
\phi^{\prime \prime}(t) \phi(t)-(1+\gamma) \phi^{\prime}(t)^{2} \geq-2(1+2 \gamma)(E(0)+\beta) \phi(t) .
$$

We consider three different cases for the sign of the initial energy $E(0)$ :

(1) If $E(0)<0$, we choose $\beta=-E(0)$, then (4.11) becomes

$$
\phi^{\prime \prime}(t) \phi(t)-(1+\gamma) \phi^{\prime}(t)^{2} \geq 0
$$

If we choose $T_{0}$ and $t_{0}$ such that $\frac{\phi(0)}{\gamma \phi^{\prime}(0)} \leq T_{0}$, by considering the assumption (1), Lemma 4.1 implies that $\phi(t)$ tends to infinity as

$$
t \rightarrow t_{1} \leq \frac{\phi(0)}{\gamma \phi^{\prime}(0)}=\frac{\left\|u_{0}\right\|^{2}+T_{0}\left\|u_{0 x}\right\|^{2}+T_{0}\left\|u_{0 x x}\right\|^{2}-E(0) t_{0}^{2}}{\gamma\left[\int_{\Omega} 2 u_{0}(x) u_{1}(x) d x-E(0) t_{0}\right]} .
$$

This is a contradiction to the fact that the maximal time of the existence of the solution is infinite. Thus, we can conclude that the solution of problem (1.1)-(1.3) blows up in finite time.

To complete the proof, we need to determine that $T_{0}$ and $t_{0}$ are positive constants. We choose $t_{0}$ large enough so that

$$
2\left(u_{0}, u_{1}\right)-E(0) t_{0}-\frac{1}{\gamma}\left(\left\|u_{0 x x}\right\|^{2}+\left\|u_{0 x}\right\|^{2}\right)>0 .
$$

Moreover, $\frac{\phi(0)}{\gamma \phi^{\prime}(0)} \leq T_{0}$ if and only if

$$
\frac{\left\|u_{0}\right\|^{2}-E(0) t_{0}^{2}}{\gamma\left[\int_{\Omega} 2 u_{0}(x) u_{1}(x) d x-E(0) t_{0}\right]-\left\|u_{0 x x}\right\|^{2}-\left\|u_{0 x}\right\|^{2}} \leq T_{0} .
$$

Clearly, $T_{0}$ and $t_{0}$ are positive constants.

(2) If $E(0)=0$, we choose $\beta=0$ and $T_{0}$ such that

$$
\frac{\left\|u_{0}\right\|^{2}}{2 \gamma\left[\int_{\Omega} u_{0}(x) u_{1}(x) d x\right]-\left\|u_{0 x x}\right\|^{2}-\left\|u_{0 x}\right\|^{2}} \leq T_{0} .
$$


Then (4.11) becomes

$$
\phi^{\prime \prime}(t) \phi(t)-(1+\gamma) \phi^{\prime}(t)^{2} \geq 0
$$

By considering assumption (2), Lemma 4.1 implies that $\phi(t)$ tends to infinity as

$$
t \rightarrow t_{2} \leq \frac{\left\|u_{0}\right\|^{2}+T_{0}\left\|u_{0 x}\right\|^{2}+T_{0}\left\|u_{0 x x}\right\|^{2}}{2 \gamma \int_{\Omega} u_{0}(x) u_{1}(x) d x}
$$

This is a contradiction to the fact that the maximal time of the existence of the solution is infinite. Thus, we can conclude that the solution of problem (1.1)-(1.3) blows up in finite time.

(3) If $E(0)>0$, we choose $\beta=0$, then (4.11) becomes

$$
\phi^{\prime \prime}(t) \phi(t)-(1+\gamma) \phi^{\prime}(t)^{2} \geq-2(1+2 \gamma) E(0) \phi(t) .
$$

We now define the auxiliary function $J(t)$ as follows:

$$
J(t)=\phi^{-\gamma}(t)
$$

Now we compute

$$
J^{\prime}(t)=-\gamma \phi^{-\gamma-1}(t) \phi^{\prime}(t)
$$

and

$$
\begin{aligned}
J^{\prime \prime}(t) & =\gamma(\gamma+1) \phi^{-\gamma-2}(t) \phi^{\prime}(t)^{2}-\gamma \phi^{-\gamma-1}(t) \phi^{\prime \prime}(t) \\
& =-\gamma \phi^{-\gamma-2}(t)\left[\phi^{\prime \prime}(t) \phi(t)-(1+\gamma) \phi^{\prime}(t)^{2}\right] \\
& \leq 2 \gamma(1+2 \gamma) E(0) \phi^{-\gamma-1}(t) .
\end{aligned}
$$

By considering assumption (3), we deduce

$$
J^{\prime}(0)=-\gamma \phi^{-\gamma-1}(0) \phi^{\prime}(0)<0 .
$$

Now let

$$
t^{*}=\sup \left\{\tau \mid J^{\prime}(\tau)<0, \tau \in[0, t)\right\}
$$

Because of the continuity of $J^{\prime}(t), t^{*}$ is positive, so multiplying (4.17) by $2 J^{\prime}(t)$, we obtain

$$
\begin{aligned}
\frac{d}{d t}\left[J^{\prime}(t)\right]^{2} & \geq 4 \gamma(2 \gamma+1) E(0) \phi^{-\gamma-1}(t) J^{\prime}(t) \\
& =-4 \gamma^{2}(2 \gamma+1) E(0) \phi^{-2 \gamma-2}(t) \phi^{\prime}(t) \\
& =4 \gamma^{2} E(0) \frac{d}{d t}\left[\phi^{-2 \gamma-1}(t)\right], \quad \forall t \in\left[0, t^{*}\right) .
\end{aligned}
$$


Integrating (4.18) over $[0, t)$, we obtain

$$
\begin{aligned}
J^{\prime}(t)^{2} & \geq J^{\prime}(0)^{2}+4 \gamma^{2} E(0) \phi^{-2 \gamma-1}(t)-4 \gamma^{2} E(0) \phi^{-2 \gamma-1}(0) \\
& \geq J^{\prime}(0)^{2}-4 \gamma^{2} E(0) \phi^{-2 \gamma-1}(0)
\end{aligned}
$$

From assumption (3), we obtain

$$
J^{\prime}(0)^{2}-4 \gamma^{2} E(0) \phi^{-2 \gamma-1}(0)>0 \text {. }
$$

Hence, making use of the continuity of $J^{\prime}(t)$, we obtain

$$
J^{\prime}(t) \leq-\left[J^{\prime}(0)^{2}-4 \gamma^{2} E(0) \phi^{-2 \gamma-1}(0)\right]^{\frac{1}{2}}, \quad \forall t \in\left[0, t^{*}\right) .
$$

Integrating (4.20) from 0 to $t$ leads to

$$
J(t) \leq J(0)-\left[J^{\prime}(0)^{2}-4 \gamma^{2} E(0) \phi^{-2 \gamma-1}(0)\right]^{\frac{1}{2}} t, \quad \forall t>0 .
$$

We choose $T_{0}$ such that

$$
\frac{J(0)}{\left.U^{\prime}(0)^{2}-4 \gamma^{2} E(0) \phi^{-2 \gamma-1}(0)\right]^{\frac{1}{2}}} \leq T_{0}
$$

Then there exists a finite positive number $t_{3}$ such that $J\left(t_{3}\right)=0$ and $0<t_{3} \leq T^{*}=$ $\frac{J(0)}{\left.U^{\prime}(0)^{2}-4 \gamma^{2} E(0) \phi^{-2 \gamma-1}(0)\right]^{\frac{1}{2}}}$. Thus, $\phi(t) \rightarrow \infty$ as $t \rightarrow t_{3}$. This is a contradiction to the fact that the maximal time of the existence of the solution is infinite. The theorem is proved.

Example 4.1 For the initial boundary value problem (1.1)-(1.3), we take specific functions $g(s), u_{0}(x)$ and $u_{1}(x)$ satisfying the conditions of Theorem 2.2. We first discuss:

(i) The case $E(0)<0$. To this end, we take $u_{0}(x)=\sin ^{2} \pi x, u_{1}(x)=1$ and $g(s)=-s$. Obviously, $u_{0}(x) \in H^{2}(0,1), u_{1}(x) \in L^{2}(0,1), g(0)=0, G\left(u_{0 x x}\right) \in L^{1}(0,1), s g(s)=-s^{2}$ and $2(1+2 \gamma) G(s)=-\frac{2(1+2 \gamma)}{2} s^{2}$. Thus, when $\gamma=1, g(s)$ satisfies assumption (2.1) of Theorem 2.2. After some simple calculation, we get $\left\|u_{1}\right\|^{2}=1$, $\left\|u_{0 x}\right\|^{2}=\frac{\pi^{2}}{2},\left\|u_{0 x x}\right\|^{2}=2 \pi^{2}, \int_{0}^{1} G\left(u_{0 x x}\right) d x=\pi^{4}$ and

$$
E(0)=\left\|u_{1}\right\|^{2}+\left\|u_{0 x x}\right\|^{2}-2 \int_{\Omega} G\left(u_{0 x x}\right) d x=1+2 \pi^{2}-2 \pi^{4} .
$$

Hence, we see from (4.22) that $E(0)<0$. We choose $t_{0}=10$ so that

$$
2\left(u_{0}, u_{1}\right)-E(0) t_{0}-\frac{1}{\gamma}\left(\left\|u_{0 x x}\right\|^{2}+\left\|u_{0 x}\right\|^{2}\right)=1+20 \pi^{4}-\frac{21}{2} \pi^{2}-10>0 .
$$

Then the conditions of Theorem 2.2 are satisfied. Hence there exists a $t_{1} \leq \frac{\phi(0)}{\gamma \phi^{\prime}(0)}$ such that $\phi(t) \rightarrow \infty$ as $t \rightarrow t_{1}^{-}$.

(ii) The case $E(0)=0$. We take $u_{0}(x)=\sin ^{2} \pi x, u_{1}(x)=k_{1}$ and $g(s)=k_{2} s$. Then $u_{0}(x) \in H^{2}(0,1), u_{1}(x) \in L^{2}(0,1), G\left(u_{0 x x}\right) \in L^{1}(0,1)$, and, when $\gamma=1, g(s)$ satisfies assumption (2.1) of Theorem 2.2, and from above relations we obtain that 
$\left\|u_{1}\right\|^{2}=k_{1}^{2},\left\|u_{0 x}\right\|^{2}=\frac{\pi^{2}}{2},\left\|u_{0 x x}\right\|^{2}=2 \pi^{2}, \int_{0}^{1} G\left(u_{0 x x}\right) d x=k_{2} \pi^{4}$ as well as, when $k_{1}=50$ and $k_{2}=\frac{1250+\pi^{2}}{\pi^{4}}$,

$$
E(0)=\left\|u_{1}\right\|^{2}+\left\|u_{0 x x}\right\|^{2}-2 \int_{\Omega} G\left(u_{0 x x}\right) d x=k_{1}^{2}+2 \pi^{2}-2 k_{2} \pi^{4}=0
$$

and

$$
2\left(u_{0}, u_{1}\right)-\frac{1}{\gamma}\left(\left\|u_{0 x x}\right\|^{2}+\left\|u_{0 x}\right\|^{2}\right)=50-\frac{5}{2} \pi^{2}>0 .
$$

Then the conditions of Theorem 2.2 are satisfied. Hence there exists a $t_{2} \leq \frac{\phi(0)}{\gamma \phi^{\prime}(0)}$ such that $\phi(t) \rightarrow \infty$ as $t \rightarrow t_{2}^{-}$.

(iii) The case $E(0)>0$. Now we take $u_{0}(x)=\sin ^{2} \pi x, u_{1}(x)=100$ and $g(s)=k_{3} s$. When $\gamma=\frac{1}{4}, g(s)$ satisfies assumption (2.1) of Theorem 2.2. Then $\left\|u_{0}\right\|^{2}=\frac{1}{2},\left\|u_{1}\right\|^{2}=10^{4}$, $\left\|u_{0 x}\right\|^{2}=\frac{\pi^{2}}{2},\left\|u_{0 x x}\right\|^{2}=2 \pi^{2}, \int_{0}^{1} G\left(u_{0 x x}\right) d x=k_{3} \pi^{4}$, and we choose $k_{3}=51$ so that

$$
E(0)=\left\|u_{1}\right\|^{2}+\left\|u_{0 x x}\right\|^{2}-2 \int_{\Omega} G\left(u_{0 x x}\right) d x=10^{4}+2 \pi^{2}-2 k_{3} \pi^{4}>0
$$

Taking $T_{0}=\frac{1}{10}$ gives

$$
\left(u_{0}, u_{1}\right)>\left[E(0)\left(\left\|u_{0}\right\|^{2}+\frac{1}{10}\left\|u_{0 x}\right\|^{2}+\left\|u_{0 x x}\right\|^{2}\right)\right]^{\frac{1}{2}}
$$

Then the conditions of Theorem 2.2 are satisfied. Hence there exists a finite positive number $t_{3}$ such that $J\left(t_{3}\right)=0$ and $0<t_{3} \leq T^{*}=\frac{J(0)}{\left[J^{\prime}(0)^{2}-4 \gamma^{2} E(0) \phi^{-2 \gamma-1}(0)\right]^{\frac{1}{2}}}$.

Thus, $\phi(t) \rightarrow \infty$ as $t \rightarrow t_{3}$.

\section{Energy decay estimate for a solution}

In this section, we study the energy decay estimates for the solution for the problem (1.1)(1.3). In order to prove our result, we need the following basic lemma, which can be found in $[25]$.

Lemma 5.1 ([25]) Let $F: \mathbb{R}^{+} \rightarrow \mathbb{R}^{+}\left(\mathbb{R}^{+}=[0, \infty)\right)$ be a non-increasing function and assume that there exists a constant $M>0$ such that

$$
\int_{t}^{\infty} F(s) d s \leq M F(t), \quad \forall t \in \mathbb{R}^{+} .
$$

Then

$$
F(t) \leq F(0) e^{1-\frac{t}{M}}, \quad \forall t \geq 0
$$

Proof of Theorem 2.3 Let the energy be

$$
\begin{aligned}
E_{1}(t) & =\left\|u_{t}(t)\right\|^{2}+\left\|u_{x x}(t)\right\|^{2}-2 \int_{0}^{1} G\left(u_{x x}\right) d x \\
& =2 \int_{0}^{t}\left(\left(u_{x x t}, u_{t}\right)-\left(u_{x x x x t}, u_{t}\right)\right) d \tau+\left\|u_{1}\right\|^{2}+\left\|u_{0 x x}\right\|^{2}
\end{aligned}
$$




$$
\begin{aligned}
& -2 \int_{0}^{1} G\left(u_{0 x x}\right) d x \\
= & 2 \int_{0}^{t}\left(-\left\|u_{x t}(\tau)\right\|^{2}-\left\|u_{x x t}(\tau)\right\|^{2}\right) d \tau+\left\|u_{1}\right\|^{2}+\left\|u_{0 x x}\right\|^{2} \\
& -2 \int_{0}^{1} G\left(u_{0 x x}\right) d x
\end{aligned}
$$

for any global solution of the problem (1.1)-(1.3). A simple computation gives

$$
\begin{aligned}
E_{1}(S)-E_{1}(T)= & -2 \int_{0}^{S}\left\|u_{x t}(t)\right\|^{2} d t-2 \int_{0}^{S}\left\|u_{x x t}(t)\right\|^{2} d t \\
& +2 \int_{0}^{T}\left\|u_{x t}(t)\right\|^{2} d t+2 \int_{0}^{T}\left\|u_{x x t}(t)\right\|^{2} d t \\
= & 2 \int_{S}^{T}\left\|u_{x t}(t)\right\|^{2} d t+2 \int_{S}^{T}\left\|u_{x x t}(t)\right\|^{2} d t, \quad \forall 0 \leq S \leq T<\infty .
\end{aligned}
$$

From (5.3) and assumption (1), it follows that the energy $E_{1}(t)$ is non-increasing and $E_{1}(t) \geq 0$. Multiplying both sides of (1.1) by $u(x, t)$, integrating over $(S, T) \times \Omega$, and applying integration by parts, we obtain

$$
\begin{aligned}
\int_{S}^{T} & \int_{0}^{1}-u_{t}^{2}+u_{x x}^{2} d x d t \\
= & -\left[\int_{0}^{1} u u_{t} d x\right]_{S}^{T}-\frac{1}{2}\left[\int_{0}^{1} u_{x}^{2} d x\right]_{S}^{T}-\frac{1}{2}\left[\int_{0}^{1} u_{x x}^{2} d x\right]_{S}^{T} \\
& +\int_{S}^{T} \int_{0}^{1} g\left(u_{x x}\right) u_{x x} d x d t, \quad \forall 0 \leq S<T<\infty .
\end{aligned}
$$

Now adding $2 \int_{S}^{T} \int_{0}^{1}\left(u_{t}^{2}-G\left(u_{x x}\right)\right) d x d t$ to both sides of (5.4), we obtain

$$
\begin{aligned}
\int_{S}^{T} E_{1}(t) d t= & \int_{S}^{T} \int_{0}^{1} 2 u_{t}^{2} d x d t-\left[\int_{0}^{1} u u_{t} d x\right]_{S}^{T} \\
& -\frac{1}{2}\left[\int_{0}^{1} u_{x}^{2} d x\right]_{S}^{T}-\frac{1}{2}\left[\int_{0}^{1} u_{x x}^{2} d x\right]_{S}^{T} \\
& +\int_{S}^{T} \int_{0}^{1}\left[g\left(u_{x x}\right) u_{x x}-2 G\left(u_{x x}\right)\right] d x d t .
\end{aligned}
$$

From (5.5) and assumption (2), we deduce

$$
\begin{aligned}
\int_{S}^{T} E_{1}(t) d t & \leq \int_{S}^{T} \int_{0}^{1} 2 u_{t}^{2} d x d t-\left[\int_{0}^{1} u u_{t} d x\right]_{S}^{T}-\frac{1}{2}\left[\int_{0}^{1} u_{x}^{2} d x\right]_{S}^{T}-\frac{1}{2}\left[\int_{0}^{1} u_{x x}^{2} d x\right]_{S}^{T} \\
& =\int_{S}^{T} \int_{0}^{1} 2 u_{t}^{2} d x d t+\left[\int_{0}^{1} u u_{t} d x\right]_{T}^{S}+\frac{1}{2}\left[\int_{0}^{1} u_{x}^{2} d x\right]_{T}^{S}+\frac{1}{2}\left[\int_{0}^{1} u_{x x}^{2} d x\right]_{T}^{S} \\
& =\int_{S}^{T} \int_{0}^{1} 2 u_{t}^{2} d x d t+\left(\int_{0}^{1} u u_{t} d x\right)(S)+\frac{1}{2}\left(\int_{0}^{1} u_{x}^{2} d x\right)(S) \\
& +\frac{1}{2}\left(\int_{0}^{1} u_{x x}^{2} d x\right)(S)
\end{aligned}
$$




$$
\begin{aligned}
& -\left(\int_{0}^{1} u u_{t} d x\right)(T)-\frac{1}{2}\left(\int_{0}^{1} u_{x}^{2} d x\right)(T)-\frac{1}{2}\left(\int_{0}^{1} u_{x x}^{2} d x\right)(T) \\
\leq & \int_{S}^{T} \int_{0}^{1} 2 u_{t}^{2} d x d t+\left(\int_{0}^{1} u u_{t} d x\right)(S)+\frac{1}{2}\left(\int_{0}^{1} u_{x}^{2} d x\right)(S) \\
& +\frac{1}{2}\left(\int_{0}^{1} u_{x x}^{2} d x\right)(S)-\left(\int_{0}^{1} u u_{t} d x\right)(T) .
\end{aligned}
$$

Making use of the non-increasingness property of $E_{1}(t)$ and the Poincaré inequality, we obtain

$$
2 \int_{S}^{T} \int_{0}^{1} u_{t}^{2} d x d t \leq 2 \int_{S}^{T} \int_{0}^{1} u_{x t}^{2} d x d t \leq E_{1}(S)-E_{1}(T) \leq E_{1}(S) .
$$

Using the Poincaré inequality and integrating by parts, we see that

$$
\begin{aligned}
\int_{0}^{1} u^{2}(x, t) d x & \leq \int_{0}^{1} u_{x}^{2}(x, t) d x=-\int_{0}^{1} u(x, t) u_{x x}(x, t) d x \\
& \leq \frac{1}{2} \int_{0}^{1} u^{2}(x, t)+u_{x x}^{2}(x, t) d x
\end{aligned}
$$

Therefore,

$$
\int_{0}^{1} u^{2}(x, t) d x \leq \int_{0}^{1} u_{x x}^{2}(x, t) d x
$$

Using the Cauchy inequality and (5.9), we have

$$
\begin{aligned}
& \left|\int_{0}^{1} u u_{t} d x\right| \leq \frac{1}{2} \int_{0}^{1}\left(u^{2}+u_{t}^{2}\right) d x \leq \frac{1}{2} \int_{0}^{1}\left(u_{x x}^{2}+u_{t}^{2}\right) d x \leq \frac{1}{2} E_{1}(t) \\
& \frac{1}{2} \int_{0}^{1} u_{x}^{2} d x \leq \frac{1}{2} \int_{0}^{1} u_{x x}^{2} d x \leq \frac{1}{2} E_{1}(t) .
\end{aligned}
$$

Now, from (5.7)-(5.11), it follows that

$$
\int_{S}^{T} E_{1}(t) d t \leq 3 E_{1}(S), \quad 0 \leq S<T<\infty
$$

Letting $T \rightarrow \infty$, we deduce from (5.12) that

$$
\int_{S}^{\infty} E_{1}(t) d t \leq 3 E_{1}(S), \quad \forall S \geq 0
$$

and now conclude from Lemma 5.1 that

$$
E_{1}(t) \leq E_{1}(0) e^{1-\frac{t}{3}}, \quad \forall S \geq 0 .
$$

This completes the proof. 


\section{Discussion and conclusions}

\subsection{Discussion}

Theorem 2.3 uses the conditions of Theorem 2.1, that is, it holds under Assumption 2.1 and the assumption

$$
s g(s) \leq 2 G(s) \leq 0 \quad \text { for all } s \in \mathbb{R}
$$

Problem (1.1)-(1.3) admits a global weak solution in the space $X(T)$, and the solution decays with an exponential rate. At the same time, Theorem 2.2 requires the energy identity $E(t)=E(0)$ and the assumption

$$
s g(s) \geq 2(1+2 \gamma) G(s), \quad s \in \mathbb{R} .
$$

Then problem (1.1)-(1.3) has no global solution in the space

$$
C\left([0, T] ; H^{2}(0,1)\right) \cap C^{1}\left([0, T] ; L^{2}(0,1)\right) \cap H^{1}\left([0, T] ; H^{2}(0,1)\right) .
$$

Thus the conclusions of Theorems 2.3 and 2.2 are compatible. In this paper, we just consider the problems in a 1-dimensional space, but it would be interesting to investigate whether Eq. (1.1) has a global solution to the Cauchy problem or the initial boundary value problem in a high-dimensional space and for other functional spaces. This question is interesting and still open.

\subsection{Conclusions}

The goal of this paper is to study the existence and nonexistence of global weak solutions to the initial boundary value problem for a nonlinear beam equation with double damping terms

$$
u_{t t}-u_{x x t}+u_{x x x x}+u_{x x x x t}=g\left(u_{x x}\right)_{x x}, \quad x \in \Omega, t>0
$$

where $g(s)$ is a given nonlinear function. When $u_{0} \in H, u_{1} \in L^{2}$ and Assumption 2.1 holds, for any $T>0$, the problem (1.1)-(1.3) admits a unique global weak solution $u \in X(T)$. And the decay estimate of the energy functional for the global solution is given by making use of a differential inequality technique. Moreover, when $u_{0} \in H^{2}(\Omega), u_{1} \in L^{2}(\Omega), G\left(u_{0 x}\right) \in$ $L^{1}(\Omega)$ satisfy certain conditions, the solution to problem (1.1)-(1.3) blows up in finite time.

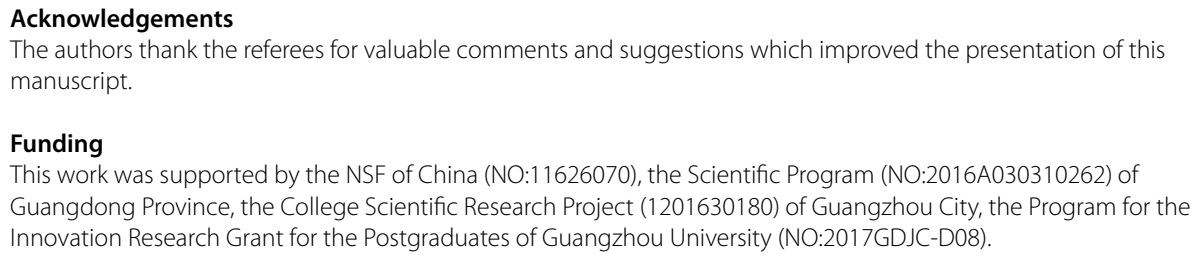

Abbreviations

Not applicable.

Availability of data and materials

Not applicable. 


\section{Competing interests}

The authors declare that they have no competing interests.

\section{Authors' contributions}

Each of the authors contributed to each part of this study equally. All authors read and approved the final version of the manuscript.

\section{Publisher's Note}

Springer Nature remains neutral with regard to jurisdictional claims in published maps and institutional affiliations.

Received: 26 March 2018 Accepted: 13 September 2018 Published online: 24 September 2018

\section{References}

1. Banks, H.T., Gilliam, D.S., Shubov, V.I: Global solvability for damped abstract nonlinear hyperbolic systems. Differ. Integral Equ. 10, 309-332 (1997)

2. Chen, G., Lu, B.: The initial-boundary value problems for a class of nonlinear wave equations with damping term. J. Math. Anal. Appl. 351, 1-15 (2009)

3. Ackleh, A.S., Banks, H.T., Pinter, G.A.: A nonlinear beam equation. Appl. Math. Lett. 15(3), 381-387 (2002)

4. Banks, H.T., Gilliam, D.S., Shubov, V.I.: Well-posedness for a one dimensional nonlinear beam. In: Computation and Control IV, Progress in Systems and Control Theory, vol. 20, pp. 1-21. Birkhäuser, Boston (1995)

5. Chen, G.: Initial boundary value problem for a damped nonlinear hyperbolic equation. J. Partial Differ. Equ. 16(1), 49-61 (2003)

6. Song, C., Yang, Z.: Existence and nonexistence of global solutions to the Cauchy problem for a nonlinear beam equation. Math. Methods Appl. Sci. 33, 563-575 (2010)

7. Yu, J., Shang, Y., Di, H.: Existence and nonexistence of global solutions to the Cauchy problem of the nonlinear hyperbolic equation with damping term. AIMS Math. (accepted) (2018)

8. Chen, G., Wang, Y., Zhao, Z.: Blow-up of solution of an initial boundary value problem for a damped nonlinear hyperbolic equation. Appl. Math. Lett. 17, 491-497 (2004)

9. Aassila, M., Guesmia, A.: Energy decay for a damped nonlinear hyperbolic equation. Appl. Math. Lett. 12, 49-52 (1999)

10. Yang, Z:: Global existence asymptotic behavior and blowup of solutions for a class of nonlinear wave equations with dissipative term. J. Differ. Equ. 187, 520-540 (2003)

11. Song, C.: Nonexistence of global solutions of nonlinear hyperbolic equation with material damping. Appl. Math. Mech. 27(7), 975-981 (2006)

12. Chen, G., Da, F.: Blow-up of solution of Cauchy problem for three-dimensional damped nonlinear hyperbolic equation. Nonlinear Anal. 71, 358-372 (2009)

13. Xu, R., Wang, S., Yang, Y.: Initial boundary value problem for a class of fourth-order wave equation with viscous damping term. Appl. Anal. 92, 1403-1416 (2013)

14. Liu, W., Chen, K.: Existence and general decay for nondissipative hyperbolic differential inclusions with acoustic/memory boundary conditions. Math. Nachr. 289, 300-320 (2016)

15. Di, H., Shang, Y.: Blow-up phenomena for a class of metaparabolic equations with time dependent coefficient. AlMS Ser. Appl. Math. 2(4), 647-657 (2017)

16. Zhao, W., Liu, W.: A note on blow-up of solutions for a class of fourth-order wave equation with viscous damping term. Appl. Anal. (2017) In press. https://doi.org/10.1080/00036811.2017.313410

17. Liu, W., Sun, Y., Li, G.: On decay and blow-up of solutions for a singular nonlocal viscoelastic problem with a nonlinear source term. Topol. Methods Nonlinear Anal. 49(1), 299-323 (2017)

18. Liu, W., Yu, J.: A note on blow-up of solution for a class of semilinear pseudo-parabolic equations. J. Funct. Anal. 274(5), 1276-1283 (2018)

19. Sun, F., Liu, L., Wu, Y.: Finite time blow-up for a class of parabolic or pseudo-parabolic equations. Comput. Math. Appl. 75(10), 3685-3701 (2018)

20. Xu, R., Liu, J., et al.: Asymptotic behaviour of solution for multidimensional viscoelasticity equation with nonlinear source term. Bound. Value Probl. 2013, 42 (2013)

21. Khelghati, A., Baghaei, K.: Blow-up phenomena for a class of fourth-order nonlinear wave equations with a viscous damping term. Math. Methods Appl. Sci. 41(2), 490-494 (2018)

22. Dautray, R., Lions, J.L.: Mathematical Analysis and Numerical Methods for Science and Technology, Volume 5 , Evolution Problems I. Springer, New York (1993)

23. Levine, H.A.: Some additional remarks on the nonexistence of global solutions to nonlinear wave equations. SIAM J. Math. Anal. 5, 138-146 (1974)

24. Levine, H.A.: Instability and nonexistence of global solutions of nonlinear wave equation of the form $P u_{t t}=A u+F(u)$. Trans. Am. Math. Soc. 192, 1-21 (1974)

25. Komornik, V.: Exact Controllability and Stabilization: Multiplier Method. Wiley, Paris (1994) 